\title{
Can We Change a Look at Atherosclerotic Aortic Aneurism Treatment?
}

\author{
Vladimir Petrovich Krylov, Alexander Gennadyevich Mrochek1, Leonid Petrovich Titov', \\ Valentina Nikolaevna Gaiduk1, Leonid Ivanovich Reut', Aleksey Leonidovich Smaliakou' \\ ${ }^{1}$ Republican Scientific and Practical Centre "Cardiology", Ministry of Health of Belarus, Minsk, Belarus \\ ${ }^{2}$ Republican Scientific and Practical Centre "Epidemiology and Microbiology", Ministry of Health of Belarus, \\ Minsk, Belarus \\ Email: krylov v p@mail.ru, a.mrochek@mail.by, leotit310@gmail.com
}

Received 26 April 2014; revised 28 May 2014; accepted 4 June 2014

Copyright @ 2014 by authors and Scientific Research Publishing Inc.

This work is licensed under the Creative Commons Attribution International License (CC BY).

http://creativecommons.org/licenses/by/4.0/

(c) $\underset{\mathrm{EY}}{\mathrm{B}}$ Open Access

\section{Abstract}

In recent years, the increasing number of cardiologists and cardiac surgeons tend to think that surgical treatment of patients with atherosclerotic aneurisms does not fully comply with contemporary ideas of what the disease is. Some data show that early operations in the presence of this pathology are associated with an unreasonably high mortality. Additionally, the use of intra-aortic stents and grafts cannot principally affect the therapeutic efficacy. Therefore, more attention is paid to the development of conservative therapeutic approaches leaving surgery defeated without surgical treatment. Two groups of patients with similar descending thoracic aortic atherosclerotic aneurisms (DTAAA) and abdominal aortic aneurisms (AAA) were retro- and prospectively studied over a 2-year period. Control group (Comparison group), (63 patients) received common surgical treatment from 2009 to 2010 whereas Main group (121 subjects) received multifaceted medical treatment to remove inflammatory reactions, strengthen aortic wall and control its dilation from 2011 to 2012. Operative treatment was used only in case of potential aneurism rupture. The comparison of the two groups of subjects showed that 2-year all-cause mortality in control group was $20.6 \%$ while in the main group it amounted to $9.1 \%$ due to the similar incidence of aneurism ruptures and deaths associated with concomitant diseases. It suggests that the odds ratio (OR) of survival when using attenuated therapeutic approach to treating atherosclerotic aneurisms is 2.6fold higher compared to conventional surgical approach. One of the principal factors contributing to a higher mortality when using traditional surgical approach was the presence of polyorgan pathology that required constant medical correction irrespective of therapeutic option (surgical or medical) used. Another important factor is aortic aneurism wall frailty. The development of mechanisms that would allow its strengthening is considered a principal challenge of cutting-edge medicine that should be based on studies of triggers, molecular genetic bases of aortic wall immune-depending inflammatory formation, the production of pro-inflammatory cytokines, metalloproteinase activity that damages elastin and collagen fibers. 


\section{Keywords}

\section{Atherosclerotic Aortic Aneurisms, Surgical and Conservative Treatment}

\section{Introduction}

Based on data provided by some researchers [1] [2], detection frequency of abdominal aortic aneurisms (AAA) has increased thrice over the last 3 decades, and those of minor AAA (up to $5 \mathrm{~cm}$ in diameter) showed a 10-fold rise. The rate of thoracic aortic aneurisms amounts to six cases in 100,000 of population and keeps on rising due to the increasing life expectancy and better diagnosis [3].

In the present study, in view of similar nature of etiopathogenic processes present in aortic wall while progressing to atherosclerotic lesion, we unify terms "descending aortic thoracic and abdominal segments" to "descending aortic segments" and use the term "descending aortic aneurisms" (DAA) instead.

The development of atherosclerotic DAA is most likely determined by a combination of dysgenics with degenerative-inflammatory changes in its wall and the progression of atherosclerotic disease. Main pathogenetic factor of it is aortitis of both non-infectious and infectious nature. The latter includes the proliferation of infectious agents of bacterial and viral nature [4] [5] and the infiltration of aortic wall by the immunoinflammatory cells (monocytes, macrophages, CD4+ and CD8+ T-lymphocytes) enhancing greatly the synthesis of proteolytic enzymes (elastase, collagenase, metalloproteases-MMP2, MMP-9, catepsins and regulatory miRNA) as affected by infectious agent peptides, cytokins, and chemokins [6] [7]. These factors contributed to impaired aortic wall structural integrity and muscular layer elastic frame. In case of increased peripheral resistance in lower extremity arteries, impaired visceral branch circulation, and developed systemic hypertension, the structurally and functionally altered aortic wall becomes unable to sustain the hemodynamic load.

Treatment of DAA is still a surgical challenge, but the more profound look at this problem allows revealing their multiple somatic diseases, with cardiovascular disease for the most part. The specified factors increase even higher mortality observed in aortic prosthetic repairs up to 32\% - 60\% [8].

Endovascular treatment methods of DAA subjects represent an alternative to a conventional surgical option. The literature sources, however, reveal endless discussions on choosing treatment modalities in these populations. The EVAR-1 study investigating treatment of aneurisms in patients aged 60 years old and older with AAA diameter exceeding $5.5 \mathrm{~cm}$ over the 4-year observation period did not yield any results that showed any allcause mortality advantages of endovascular prosthetic repair prior to open surgical operation. Moreover, the mortality rate associated with aneurism was reduced by $3 \%$ in patients received endovascular treatment [9] [10]. The EVAR-2 study showed no significant differences between groups of endovascular and conservative treatment by all-cause mortality criteria predetermined by the presence of aneurism and life quality. The number of deaths due to aneurism rupture in conservative treatment group was consistent with the number of ruptures and post-operation mortality in endovascular treatment group. The author believes that the absence of differences in main parameters of EVAR-2 study can be explained by the rate of aneurism ruptures in subjects on conservative treatment [11].

Study objective: to compare 2-year survival in patients with atherosclerotic aneurisms of descending abdominal and thoracic segments in standard surgical modality and conservative treatment option to strengthen aortic wall, with the surgical correction only when there is a danger of rupture.

\section{Materials and Methods}

\section{Patient Characteristics}

A total of 184 patients with aneurisms of descending abdominal and thoracic aortic segments received treatment in RSPC "Cardiology”. A group of 63 in-patient individuals in Vascular Surgery Dpt. were Controls, and they were retrospectively analyzed in the period from 2009 to 2010 to check efficacy of standard surgical modality followed by 2-year supporting symptomatic medical correction.

The main group comprised 121 subjects with 2-year prospective study from 2011 to 2012 that addressed the efficacy of ambulatory medical correction aimed at reinforcing aortic wall and prevention of its further trans- 
formation with operative treatment only if there was a high threat of aneurism rupture. The dynamic control of this group was performed every 3 to 6 months, and included registration of well-being, aneurism size, BP, lipid spectrum, inflammatory markers, complications and co-morbidities. Conservative treatment to attain and maintain optimal BP, target cholesterol and low-density lipoproteins, reduced aortic oxidative and inflammatory processes, reinforcing of wall and stabilization of the disease was regular.

We provided an open prosthetic repair surgery or aortic endoprosthetic surgery in case of rapid progression of negative dynamics (increased aortic diameter $<5 \mathrm{~mm}$ at 6 months) and in the absence of contraindications for surgery.

\section{Methods}

1) Clinical studies, including CT-angiography, aortic angiography, coronary angiography, US scans.

2) Surgical treatment: aortic-ileal-femoral bifurcation prosthesis and endovascular stent graft implantation.

3) Medical treatment to reinforce aortic wall. Regular combination of ACE-inhibitors with amlodipine, b-blockers, and statins. Courses of macrolide antibiotics (roxithromycin, azithromycin) in case of emergent inflammatory process (by CRP) and symptomatic therapy.

4) Statistical methods. Calculation of probability, significance, and their values to analyze biological data [12]. Calculation method of OR—odds ratio [13].

\section{Results}

The study of DAA subjects showed no statistically evident differences in age, sex, aneurism diameter, including that of more than $50 \mathrm{~mm}$, their elongation, thoracic and abdominal site diameter, and the ratio of DTAAA/AAA localizations (Table 1). However, Main group showed an absolute number of patients with $\mathrm{d}>50 \mathrm{~mm}$ (some of

Table 1. The comparison of subjects of the main (treatment targeting medical reinforcing of aortic wall and dilation control) and control (common surgical method) subject groups observed in Belarusian Scientific and Practical Centre "Cardiology" over the 2-year period: 2011-2012 (Main group), and 2009-2010 (Controls).

\begin{tabular}{|c|c|c|c|c|c|}
\hline Values & \multicolumn{2}{|c|}{$\begin{array}{l}\text { Controls } \\
\mathrm{n}=63\end{array}$} & \multicolumn{2}{|c|}{$\begin{array}{l}\text { Main group } \\
\mathrm{n}=121\end{array}$} & $\mathrm{P} / \mathrm{OR}$ \\
\hline Age & \multicolumn{2}{|c|}{$62.4 \pm 0.97$ y.o. } & \multicolumn{2}{|c|}{$67 \pm 0.77$ у.о. } & $>0.05$ \\
\hline M & 60 & $95.2 \%$ & 107 & $88.4 \%$ & \\
\hline $\mathrm{F}$ & 3 & $4.76 \%$ & 14 & $11.6 \%$ & \\
\hline AAA & 52 & $82.5 \%$ & 91 & $75.2 \%$ & \\
\hline DAA & 11 & $17.5 \%$ & 30 & $24.8 \%$ & \\
\hline Aneurism $\mathrm{d}$ in $\mathrm{mm}$ & $45.9 \pm 2.3$ & & $43.13 \pm 2.1$ & & $>0.05$ \\
\hline d AAA in mm & $48.42 \pm 2.22$ & & $43.97 \pm 2.41$ & & $>0.05$ \\
\hline $\mathrm{d}$ DAA in mm & $44.45 \pm 2.03$ & & $48.88 \pm 2.64$ & & $>0.05$ \\
\hline Subjects with $\mathrm{d}<50 \mathrm{~mm}$ & 30 & $47.6 \%$ & 70 & $57.8 \%$ & $>0.05$ \\
\hline Subjects with $\mathrm{d}>50 \mathrm{~mm}$ & 33 & $52.4 \%$ & 51 & $42.1 \%$ & $>0.05$ \\
\hline Aneurism L in mm & $87.8 \pm 5.33$ & & $85.13 \pm 5.4$ & & $>0.05$ \\
\hline $\begin{array}{l}\text { Subjects operated or implanted } \\
\text { with stent-graft }\end{array}$ & $\begin{array}{l}56 \\
(6)\end{array}$ & $88.9 \%$ & $\begin{array}{l}17 \\
(8)\end{array}$ & $14.0 \%$ & $<0.001$ \\
\hline Non-operated (total) & 7 & $11.1 \%$ & 104 & $86.0 \%$ & $<0.001$ \\
\hline Of which due to condition severity & 7 & $11.1 \%$ & 29 & $24.0 \%$ & $<0.05$ \\
\hline $\begin{array}{l}\text { Post-operation long- and } \\
\text { short-term mortality }\end{array}$ & $4+4=8$ & $14.3 \%$ & 0 & 0 & $<0.001$ \\
\hline $\begin{array}{l}\text { Mortality w/o operation due to } \\
\text { aneurism rupture }\end{array}$ & 2 & $3.2 \%$ & 4 & $3.3 \%$ & $>0.05$ \\
\hline Other disease-related mortality & 3 & $4.8 \%$ & 7 & $5.8 \%$ & $>0.05$ \\
\hline Post-operation complications & 6 & $9.5 \%$ & 0 & 0 & $<0.001$ \\
\hline All-cause mortality & 13 & $20.6 \%$ & 11 & $9.1 \%$ & $\begin{array}{r}<0.05 \\
2.6\end{array}$ \\
\hline
\end{tabular}


them were $76 \mathrm{~mm}$ ) which is substantially higher (51 and 33) compared to Controls. Therefore, a statistically evident difference $(\mathrm{P}<0.05)$ between groups was registered concerning subjects that were poor candidates for operation due to severity of their disease. Thus, the main group showed more apparent pathology vs. Controls.

A statistically clear difference between groups by the proportion of operated subjects and non-operated subjects can be explained by the use of different modalities, and the long- and short-term mortality rates can be related to a long and focused pretreatment preparation in the main group.

In the control group consisting of 63 subjects, 56 received surgeries, 4 of which died in early post-operation period, and 4 more subjects died in a long-term period, amounting to $12.5 \%$. Six of those operated $(9.5 \%)$ did not attain better life quality. Two out of seven non-operated subjects and two subjects survived. Three died due to diseases not related with aortic aneurism and two subjects died of its rupture. All-cause mortality at 2 years was $20.6 \%$.

In general, no cases of mortality or complications related with operative treatment were noted in this group. In medical treatment of this category during at 2 years, aortic rupture was noted in 4 subjects (3.3\%). Seven more subjects (5.8\%) died of co-morbidities (myocardial infarction, stroke, chronic renal failure, cancer disease etc.), one of which had a minor aneurism. All-cause mortality was $9.1 \%$. Thus, only 17 DAA subjects of the main group received operation (14\%) at 2 years, and 104 (86.0\%) were treated conservatively. Twenty-three individuals $(22.1 \%)$ were not operated due to higher complication risk, 70 (67.3\%) due to small size of aneurism, 5 (4.8\%) due to operation refute, and poor compliance, and $1(0.96 \%)$ due to borderline age. Three individuals were prepared for stenting, and two subjects were prepared for aortic-iliac-femoral prosthesis surgery.

\section{Discussion}

The groups with DAA are similar in principal characteristics (sex, age, degree of aortic damage and its diameters) as they have similar pathology and predisposing factors. This uniformity related to aneurism rupture mortality and all-cause mortality is still observed 2 years after treatment.

The former one was $14.3 \%$ at 2 years for the Controls, while in the same period it was absent in the main group. Additionally, 6 operated control subjects (9.5\%) did not attain any substantial medical and social rehabilitation due to post-operation complications. Hence, in view of equal aneurism rupture-related mortality values and co-morbidities, all-cause mortality between two groups is significantly different: $20.6 \%$ for Controls, and $9.1 \%(\mathrm{P}<0.05)$ for the main group. This is despite the fact that the proportion of operated individuals due to condition severity was statistically larger in the main group than in control group $(\mathrm{P}<0.05)$.

Graphic comparison of some data (Figure 1) confirms, in our opinion, a high risk of operation in a majority of subjects vs. adequate conservative treatment, especially for patients with aneurism diameter less than $50 \mathrm{~mm}$.

Thus, despite our limited experience, data received from a comparative two-tear study (Table 1) suggest that the use of active surgical modality in subjects with DAA cannot be considered best option, especially in AAA

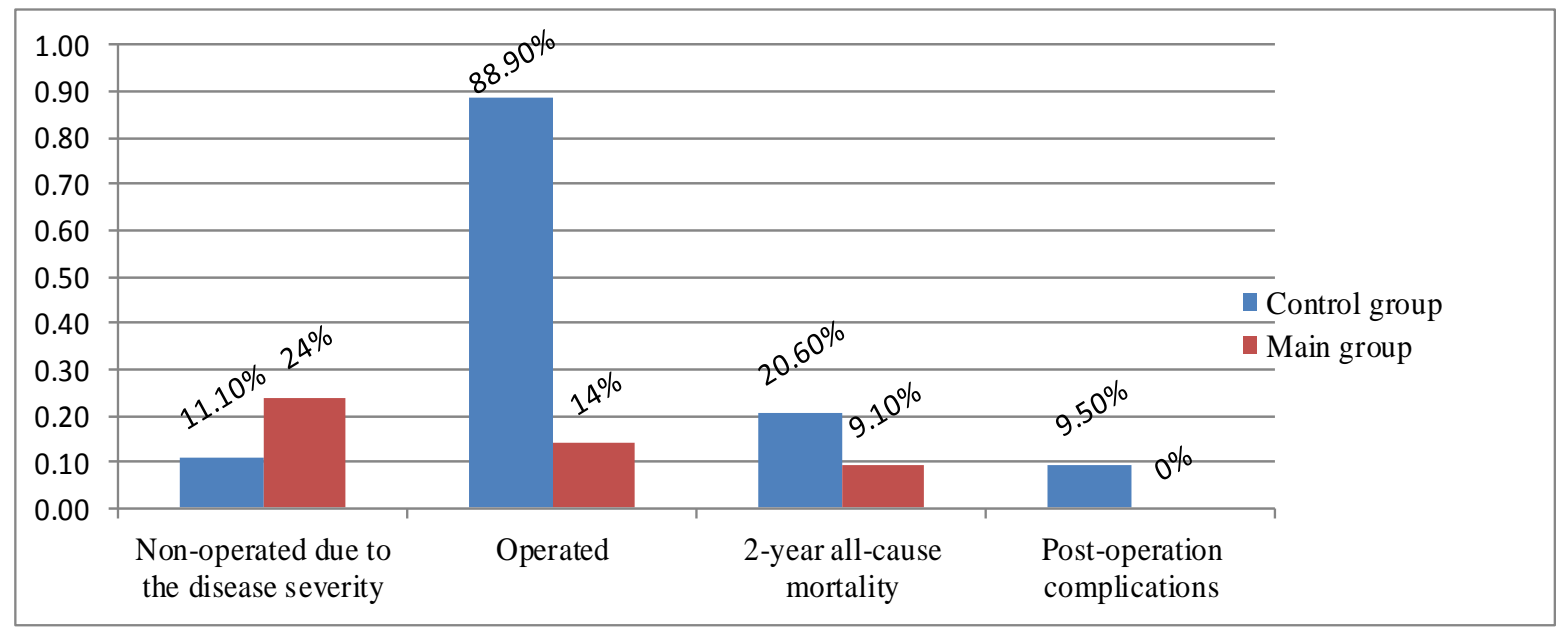

Figure 1. Group comparison. 
individuals. It seems apparent that the dynamic observation together with integrated medical treatment aimed at aortic wall reinforcement, inflammatory process reversal, BP control and concomitant pathology correction are preferred.

Based on data presented in Table 1, it is possible to determine odds ratio (OR) of 2-year mortality seen in the main group vs. control using the following method [13]: $\mathrm{OR}=13 / 11: 50 / 110=2.62$ (with non-survivors as first ratio, and survivors as second ratio). Thus, in confidence interval (CI) of 95\%, its lower and higher limit are 1.99 and 11.3 correspondingly. It suggests that mortality odds of the main groups are 2.6 times lower than in Controls. It means that survival resulting from attenuated DAA treatment is significantly higher compared to active surgical modality.

An additional benefit of medical treatment (attenuated treatment) is cost-effectiveness as the costs of operation, prosthetic materials and stent-graft implantation exceed considerably the costs required for medical treatment. Thus, adding up Controls to the main group and targeting their "symptomatic" aneurisms (51 individuals with aneurism diameter $>50 \mathrm{~mm}$ ), we see that 45 subjects must have been operated, and six subjects must have maintained on symptomatic treatment. Then the mortality noted in the main group would have been $17.4 \%$, i.e. almost the same as in the control group. Material costs (expenditures) to perform 45 operations should be added up to that. It would make at least 500,000 dollars, and when using endovascular technique it would make 50\% 80\% more expensive, i.e. 750 - 900 dollars. The comparison of expenditures on medical products is difficult, especially for patients who underwent surgery and receive symptomatic medical treatment. Thus, the expected cost effectiveness of conservative treatment with surgeries of 121 subjects for strict indications is about 1 million dollars. It once more confirms the priority of medical treatment developed by us for the main group.

A number of factors contributing to a greater survival probability in subjects receiving attenuated treatment were determined:

1) permanent medical follow-up of subjects with DAA prevents progression of the underlying disease, complications and comorbidity;

2) permanent positive emotional contact between a physician and patient enhances compliance to treatment;

3) operative treatment in the main group was provided only to psychologically and somatically prepared individuals. The applicability of considering these factors is confirmed by the fact that 6 of 11 non-survivors of the second group showed poor compliance.

\section{Conclusions}

Although, over the last few decades, a substantial surgical experience concerning DAA treatment has been obtained, there is bias concerning the appropriateness of therapeutic methods commonly used. Actually, aneurisms of any segment of descending aorta are a clear marker of generalized atherosclerotic lesion of other systems: ascending aorta, coronary, visceral, brachiocephal, ileal and lower extremity arteries, as well as of organic changes of heart and other organs. The underlying mechanism of atherosclerotic lesions is an immune-dependent inflammation with various microorganisms acting as triggers and immunocompetent cells as inducers: monocytes, macrophages, dendritic cells, neutrophils, T- and B-lymphocytes that infiltrate layers of vascular walls, produce molecules of inflammation and metalloproteases destroying elastic muscular fibers and connective tissue resulting in weakening the integrity of vascular carcass. It is a factor predisposing for the development of aneurisms which is life threatening. Thus, DAA treatment, in our opinion, should be multifaceted and systemically targeted at the key etiopathogenetic components of pathologic process: triggering factors (bacteria, viruses, and protozoa), molecular and cell components and mechanisms of the host. The principal role belongs to reduced all-cause mortality: stroke, cerebral and myocardial infarction, aortic rupture, chronic renal failure, etc. New double- or triple purpose drug products are necessary to implement this modality, but unfortunately, we hardly have any or have none.

All needed activities are being constantly developed in clinical practice. Thus, ASCOT study showed an advantage of combined ACE inhibitors with amlodipine to reduce all-cause mortality [14]. Beta-blockers hamper the increasing number of aneurisms and reduce risks of its rupture by the effect on sympathoadrenal system and then on elastin and collagen [3] [15]. The EOC recommend [15] [16] the use of statins that protect aortic wall from oxidative stress, and produce anti-inflammatory and lipid controlling effect. When inflammatory process is involved, a layer of elastin and muscular cells in the aortic wall is destroyed. Hence, macrolid antibiotic roxithromycin is recommended in this case [17]. 
Molecular genetic predisposing mechanisms contributing to aneurism formation are of interest with respect to a search of markers and predictors of their formation. Up to now, sufficient data are collected concerning association of some genes and polymorphism, proteins coded by them with secondary to human cardiovascular pathology. Certain genetic markers are associated with the increased matrix metalloproteinase activity and extracellular matrix degradation [18]. Japanese researchers [19] [20] have revealed that extracellular matrix degradation of elastin and collagen proteins are a key factor of pathogenesis of abdominal aortic aneurisms, and medical inhibition of stress-activated proteinkinase reverses extracellular matrix degradation and prevents aortic dilation [21].

Microarray analysis of global gene expression in subjects with atherosclerotic aortic aneurisms is a key issue. Although all of us have almost similar set of genes, but their polymorphism, expression profile and level considerably vary in populations as well as in certain organs and in different diseases. Microarray or biochip technique allow determination of gene hyperactivity and inhibition in subject groups, difference of their profile in healthy subjects, the effect of applied therapy seen by gene activity modulation and their correlation with pathologic process activity and therapeutic efficacy [22].

International and home studies show that DAA treatment modality is yet far from perfect. However, it is obvious that its progress is more therapeutic than surgical. Of note, not all types of DAA should be operated, but every type needs treatment. Available data confirm our suggestions on the feasibility of wait-and-see modality in patients with descending abdominal and thoracic aneurisms with dimensions of $5-5.5 \mathrm{~cm}$, Echo- and CT control and multifaceted conservative treatment, which is in line with other authors' data [23].

While investigating the clinical course of DAA at different ages and different atherosclerotic severity, we came to a conclusion about the presence of common mechanism of connective tissue destruction that manifests itself by a number of degrees of its dysplasia and genetic defects (extracellular matrix protein gene mutation and enzymes of their biosynthesis) contributing to aortic wall thinning. In addition, with the advanced age, all the above mentioned is added by atherosclerotic intima and media degeneration which tops up the formation of DAA.

If we can confirm such a mechanism of formation of atherosclerotic aneurisms, their treatment will undergo considerable changes. Approach to this challenge will be defined early diagnosis of connective tissue dysplasia, prevention of its progression at a stage of no atherosclerosis. Surgical correction of any sort in this pathology may become quite rare.

\section{References}

[1] Shirinbek, O. (2008) Infrarenal Abdominal Aneurisms: Modalities and Outcomes (Literature Review). Cardiovascular Disease Journal, 9, 50-57. (in Russian)

[2] Hallett Jr., J.W. (1992) Abdominal Aortic Aneurysm: Natural History and Treatment. Heart Disease and Stroke, 1, 303-308.

[3] Bokeria, L.A. (2010) Aortic Segment Thoracic and Thoracic Abdominal Aneurism Surgery: Guidelines for Practitioners.

[4] Titov, L.P. (2007) Infections and Modern Medical Biotechnologies. First Meeting of Scientists in Belarus. Belaruskaya Nauka, Minsk, 163-167. (in Russian)

[5] Restrepo, C.S., Jcazionez, D., Suri, R. and Vargas, D. (2011) Aortitis: Imaging Spectrum of the Infectious and Inflammatory Conditions of the Aorta. Radiographics, 31, 435-451.

[6] Harrington, D.J. (1996) Bacterial Collagenases and Collagen-Degrading Enzymes and Their Potential Role in Human Disease. Infection and Immunity, 64, 1885-1891.

[7] Milewicz, D.M. (2012) MicroRNAs, Fibrotic Remodeling, and Aortic Aneurysm. Journal Clinical Investigation, 2, 490-403. http://dx.doi.org/10.1172/JCI62204

[8] Boersma, E. (2005) Perioperative Cardiovascular Mortality in Noncardiac Surgery: Validation of the Lee Cardiac Risk Index. American Journal of Medicine, 118, 1134-1141.

[9] Greenhalgh, R.M., Brown, L.C., Kwong, G.P., et al. (2004) EVAR trial Participants. Comparison of Endovascular Aneurysm Repair with Open Repair in Patients with Abdominal Aortic Aneurysm (EVAR Trial 1), 30-Day Operative Mortality Results: Randomised Controlled Trial. Lancet, 364, 843-848. http://dx.doi.org/10.1016/S0140-6736(04)16979-1

[10] EVAR Trial Participants (2005) Endovascular Aneurysm Repair versus Open Repair in Patients with Abdominal Aor- 
tic Aneurysm (EVAR Trial 1). Randomised Controlled Trial, 365, 2179-2186.

[11] EVAR Trial Participants (2005) Endovascular Aneurysm Repair and Outcome in Patients Unfit for Open Repair of Abdominal Aortic Aneurysm (EVAR Trial 2). Randomised Controlled Trial, 365, 2187-2192.

[12] Rakitsky, P.F. (1973) Biological Statistics. Visheyshaya Shkola, Minsk, 319. (in Russian)

[13] Plavinsky, S.L. (2005) Planning, Processing and Reporting of the Results Obtained from Biomedical Studies Using SAS. Biostatistika, SPb MAPO, 559. (in Russian)

[14] Dahlof, B., Sever, P.S., Poulter, N.R., et al. (2005) ASCOT Investigators Prevention of Cardiovascular Events with an Antihypertensive Regimen of Amlodipine Adding Perindopril as Required versus Atenolol Adding Bendroflumethiazide as Required, in the Anglo-Scandinavian Cardiac Outcomes Trial-Blood Pressure Lowering Arm (ASCOTBPLA): A Multicentre Randomised Controlled Trial. Lancet, 366, 895-906. http://dx.doi.org/10.1016/S0140-6736(05)67185-1

[15] Kemma, A., John, Thomas, L.F. and Serrius Patrick, V. (2011) Cardiovascular Disease Edition. "ESC Guidelines” GEOTAR-Media, 1209-1248. (in Russian).

[16] Ejiri, J., Jnoue, N., Tsucube, T., et al. (2003) Oxidative Stress in Pathogenesis of Thoracic Aortic Aneurysm: Protective Role of Statin and Angiotensin II Type I Receptor Blocer. Cardiovascular Research, 59, 988-996. http://dx.doi.org/10.1016/S0008-6363(03)00523-6

[17] Vammen, S., Lindholt, J.S., Ostergaard, L., Fasting, H. and Henneberg, E.W. (2001) Randomized Double-Blind Controlled Trial of Roxithromycin for Prevention of Abdominal Aortic Aneurysm Expansion. British Journal of Surgery, 88, 1066-1072. http://dx.doi.org/10.1046/j.0007-1323.2001.01845.x

[18] Jeffrey, A.J., Spinale, F.G. and Ikonomidis, J.S. (2009) Transforming Growth Factor-Beta Signaling in Thoracic Aortic Aneurysm Development: A Paradox in Pathogenesis. Journal of Vascular Research, 46, 19-37.

[19] Nagasawa, A., Yoshimura, K., Suzuki, R., Mikamo, A., Yamashita, O., Ikeda, Y., Tsuchida, M. and Hamano, K.J. (2013) Important Role of the Angiotensin II Pathway in Producing Matrix Metalloproteinase-9 in Human Thoracic Aortic Aneurysms. Journal of Surgical Research, 183, 472-477. http://dx.doi.org/10.1016/j.jss.2012.12.012

[20] Yoshimura, K. and Aoki, H. (2012) Recent Advances in Pharmacotherapy Development for Abdominal Aortic Aneurysm. International Journal of Vascular Medicine, 2012, Article ID: 648167. http://dx.doi.org/10.1155/2012/648167

[21] Yoshimura, K., Aoki, H., Ikeda, Y., Furutani, A., Hamano, K. and Matsuzaki, M. (2006) Regression of Abdominal Aortic Aneurysm by Inhibition of c-Jun N-Terminal Kinase in Mice. Annals of the New York Academy of Sciences, 1085, 74-81. http://dx.doi.org/10.1196/annals.1383.031

[22] Titov, L.P. (2012). Micro-RNA: New Class of Immune Response Regulatory Molecules and Infectious Process. Up-to-Date Challenges of Human Infectious Pathology, 5, 256-261. (in Russian)

[23] Lederle, F.A., Wilson, S.E., Johnson, G.R., et al. (2002) Aneurysm Detection and Management Veterans Affairs Cooperative Study Group. Immediate Repair Compared with Surveillance of Small Abdominal Aortic Aneurysm. The New England Journal of Medicine, 346, 1437-1444. http://dx.doi.org/10.1056/NEJMoa012573 